\title{
Evidence of a critical time in constrained kinetic Ising models
}

\author{
Eduardo Follana \\ Departamento de Fisica Teorica, Facultad de Ciencias, Universidad de Zaragoza, 50009 Zaragoza, Spain \\ Felix Ritort \\ Departamento de Matematicas, Universidad Carlos III, Butarque 15, Leganés 28911, Madrid, Spain
}

(Received 4 January 1996)

\begin{abstract}
We study the relaxational dynamics of the one-spin facilitated Ising model introduced by Fredrickson and Andersen. We show the existence of a critical time which separates an initial regime in which the relaxation is exponentially fast and aging is absent from a regime in which relaxation becomes slow and aging effects are present. The presence of this fast exponential process and its associated critical time is in agreement with some recent experimental results on fragile glasses. [S0163-1829(96)04426-8]
\end{abstract}

\section{INTRODUCTION}

The subject of glassy dynamics has received a lot of attention in the last years. ${ }^{1}$ During a fast enough cooling process real glasses do reach a metastable glassy phase of free energy higher than that of the crystal phase. Apparently the glass transition behaves as a purely kinetic phenomenon and the glass does not equilibrate when probed in a time scale smaller than the relaxation time.

Laboratory experiments can measure one time extensive quantities like enthalpy and its associated specific heat and also the two-times correlation function by measuring the scattering processes. These spectra give direct information about the relaxational processes which take place in glasses. One of the most studied relaxational processes in glasses is the so-called structural or $\alpha$ relaxation which yields the structural relaxation time. While the $\alpha$ relaxation is a slow process there are other faster processes which have been observed experimentally. Close to the glass transition two fast processes have been observed: (1) the $\beta$-relaxation process predicted by the mode coupling theory, ${ }^{2}$ and observed in dielectric response measurements and (2) a faster process of the order of picoseconds observed in neutron-scattering experiments. ${ }^{3}$ In this last case, evidence has been reported on the existence of a critical time from the crossover from Debye (exponential in time and diffusive in space) to nonDebye relaxation. ${ }^{4}$ This critical time follows a temperaturedependent Arrhenius behavior. The purpose of this work is to show that the existence of this critical time is an essential ingredient of some kinetic models with short-range constrained dynamics.

Several types of models have been proposed to understand the dynamical behavior of real glasses. All of them have in common the presence of a certain type of frustration. These models can be classified in two large classes. In the first class of models, there is frustration in the energy function. During its dynamical evolution these systems move in phase space avoiding configurations of higher free energy. The dynamics can be very slow due to the existence of energy barriers (strong metastability ${ }^{5}$ ) or due to entropy barriers (strong marginality ${ }^{6}$ ). Spin glasses ${ }^{7}$ belong to these large class of systems where, in the most general case, disorder is not essential and can be self-generated by the dynamics. ${ }^{8}$

While the first class of models (at least, in the mean-field approximation) seem to capture the experimentally observed features related to the (slow) $\alpha$-relaxation process and the (fast) $\beta$-relaxation process ${ }^{12}$ it is still unclear how much they can account for this observed new type of fast Debye relaxational process. ${ }^{4}$

In the second type of models the frustration is directly introduced in the dynamics. In this case the free-energy landscape can be very simple but only certain transitions between configurations in phase space are allowed. These models are known under the name of constrained kinetic models, ${ }^{9}$ a nice example being the $n$-spin facilitated Ising model ( $n$ SFM) introduced by Fredrickson and Andersen. ${ }^{10}$

There are few studies (theoretical as well as numerical) of this simple model but we think it contains some of the fundamental processes observed in real glasses. We will study the dynamical properties of one of the simplest models belonging to the aforementioned second class. In particular we will concentrate in the 1SFM (to be defined below) at finite dimensions. We have observed that there exists a characteristic time $t_{c}$, independent of the dimensionality of the system, below which relaxation is exponential and aging is absent and above which the relaxation becomes nonexponential and aging appears. This critical time follows an Arrhenius law with the temperature and suggests a connection with some fast processes recently observed by the experimentalists. ${ }^{4}$

The paper is organized as follows. In the next section, we define the 1SFM and the main observables we are interested in. Section III contains some exact relations for one-time staggered quantities at any dimension. This reveals the existence of two fast processes. Section IV presents numerical simulations in the equilibrium regime and also in the offequilibrium regime which evidence the existence of these fast processes in the two-times correlation and integrated response function. After the conclusions we present in the Appendix the exact zero-temperature solution of the 1SFM in one dimension.

\section{THE 1SFM MODEL: DEFINITION AND OBSERVABLES}

Let us take a set of field variables $\phi(x)$ in a lattice of dimension $D$ and a Hamiltonian $H\{\phi(x)\}$. Let us consider 
an observable $O(t)$ which depends on time $t$ through the configuration of the system $O\left[\left\{\phi_{t}(x)\right\}\right]$. Now we define a discrete time dynamics for this system. In what follows we will consider a discrete Monte Carlo (MC) dynamics with random updating. A point of the lattice is randomly selected and a change of the variable $\phi(x)$ is proposed. The rate variation of $O$ in $N$ elementary moves [one Monte Carlo step (MCS)] is given by

$$
\frac{\partial O(t)}{\partial t}=\overline{P\left[\phi_{t}(x, t)\right] W\left[\phi_{t}(x, t) \rightarrow \phi_{t}^{\prime}(x, t)\right] \Delta O(t)},
$$

where $\Delta O(t)=O\left(\phi_{t}^{\prime}(x)\right)-O\left(\phi_{t}(x)\right)$ is the change in an elementary move of the set of fields $\phi_{t}(x), \overline{(\cdots)}$ stands for the average over all the possible transitions and $P\left(\phi_{t}(x)\right)$ is the probability of the configuration $\phi(x)$ at time $t$.

We consider transition probabilities $W$ of the form,

$$
\begin{aligned}
W\left[\phi_{t}(x) \rightarrow \phi_{t}^{\prime}(x)\right] \propto & \operatorname{Min}\left[\operatorname { e x p } \left\{-\beta\left[H\left(\phi_{t}^{\prime}(x)\right)\right.\right.\right. \\
& \left.\left.\left.-H\left(\phi_{t}(x)\right)\right]\right\}, 1\right] \alpha\left(\phi_{t}, \phi_{t}^{\prime}\right),
\end{aligned}
$$

where the term $\alpha\left(\phi_{t}, \phi_{t}^{\prime}\right)$ is temperature independent and cannot, in general, be absorbed in the energy function [note that the term $\alpha\left(\phi_{t}, \phi_{t}^{\prime}\right)$ can be zero for certain transitions while this can never be the case at finite temperature for the MC dynamics]. In the simplest case where $\alpha=1$ we recover the usual Metropolis algorithm for the Monte Carlo dynamics. Our purpose is to study a simple model where frustration only appears in the dynamics via the term $\alpha\left(\phi_{t}, \phi_{t}^{\prime}\right)$ due to the fact that some transitions between configurations are forbidden.

A simple model of this type is given by the $n$-spin facilitated Ising model ( $n \mathrm{SFM})$ in $D$ dimensions. ${ }^{10}$ To each node of the lattice we attach a spin variable $\sigma_{i}$ which can take the values 0,1 . The energy of the system is defined by the number of spins with value equal to one (with a minus sign), $E=-\Sigma_{i} \sigma_{i}$. By defining the new set of variables $s=2 \sigma-1$ we recognize in the previous expression the energy of an Ising paramagnet in a magnetic field $h=\frac{1}{2}$. Fredrickson and Andersen proposed a constrained dynamics for the $n$ SFM in the following way: a randomly chosen spin in the lattice is selected and a flip of that spin is proposed $\left(\sigma_{i} \rightarrow \sigma_{i}^{\prime}=1-\sigma_{i}\right)$. This change is accepted if at least $n$ of its nearest neighbors are 0 and according to the probability Min $\left(e^{-\beta \Delta E}, 1\right)$. The interest of this model is based on the fact that the nontrivial (and glassy) dynamics is all contained in the term $\alpha\left(\sigma(t), \sigma^{\prime}(t)\right)$ (note that the Hamiltonian of this model has no interaction). Consequently, the dynamics of the $n$ SFM is highly complex at infinite temperature, ${ }^{11}$ while in spin glasses and other glassy models [where $\alpha\left(\phi_{t}, \phi_{t}^{\prime}\right)=1$ ] the dynamics in this limit is trivial. The $n$ SFM model has been mainly studied in the case $n=1,2 .{ }^{10,13}$ In this work we are mainly interested in the case $n=1$ where some analytic results can be obtained.

There are several physical interpretations of the $n$ SFM. ${ }^{13}$ The simplest one relates spin variables to the local compressibility of a fluid region. In this case regions with very high compressibility can facilitate the mobility of the neighboring ones while regions of low compressibility lead to a jamming of the dynamics.

We define the set of variables $\tau_{i}=1-\sigma_{i}$. In terms of this set the transition probability $W$, Eq. (2), for the 1 SFM model reads $^{10}$

$$
W\left(\sigma_{x} \rightarrow \sigma_{x}^{\prime}\right) \propto \frac{1}{2 D} \exp \left(-\beta \sigma_{x}\right) \sum_{\mu=1}^{D}\left(\tau_{x+e_{\mu}}+\tau_{x-e_{\mu}}\right),
$$

i.e., the transition probability at point $x$ depends linearly on the magnetization of the nearest neighbors. The set $\left\{e_{\mu} ; \mu=1, \ldots, D\right\}$ is a base for the $D$-dimensional lattice. This transition probability satisfies detailed balance and is expected to generate an irreducible Markov process (in the thermodynamic limit) in case $n=1$ for any dimension at nonzero temperature (see Ref. 13 for a discussion on this point).

While the thermodynamics of this model is trivial, its dynamics is much complicated and only partial results can be obtained in some cases, especially in one dimension. In this last case, the full dynamics can be exactly solved at zero temperature. Because we are not aware of this result in the literature we present it in the Appendix. Unfortunately we have not been able to close the dynamical equations at finite temperature.

The general dynamical equation for an observable $O(t)$ in the 1 SFM model is given by

$$
\begin{aligned}
\frac{\partial O(t)}{\partial t}= & \frac{1}{2 D} \Delta O(x)\left[-e^{-\beta}+\left(1+e^{-\beta}\right) \tau_{x}\right] \\
& \times\left(\sum_{\mu=1}^{D}\left(\tau_{x+e_{\mu}}+\tau_{x-e_{\mu}}\right)\right),
\end{aligned}
$$

where $\Delta O(x)=O\left(\sigma_{x}=1\right)-O\left(\sigma_{x}=0\right)$ stands for an elementary variation of the observable $O$ at time $t$ for the change of $\sigma_{x}$ from 0 to 1 . Furthermore, the right-hand side has to be averaged over all points $x$ of the lattice.

We are interested in the energy-energy correlation function $C_{E}\left(t, t^{\prime}\right)\left(t^{\prime}<t\right)$ defined as

$$
C_{E}\left(t, t^{\prime}\right)=\frac{(1 / N) \sum_{i=1}^{N} \tau_{i}(t) \tau_{i}\left(t^{\prime}\right)-m_{\tau}\left(t^{\prime}\right) m_{\tau}(t)}{m_{\tau}\left(t^{\prime}\right)\left[1-m_{\tau}\left(t^{\prime}\right)\right]},
$$

where $m_{\tau}(t)=(1 / N) \sum_{i=1}^{N} \tau_{i}(t)$ is the global magnetization associated with the set of variables $\left\{\tau_{i} ; i=1, \ldots, N\right\} .{ }^{14} C_{E}$ is normalized in such a way that $C_{E}(t, t)=1$.

We define the staggered one-point and two-point functions

$$
\begin{gathered}
C_{0}(t)=\frac{1}{N} \sum_{i=1}^{N}(-1)^{\left(\Sigma_{\mu=1}^{D} i_{\mu}\right)} \tau_{i}, \\
C_{1}(t)=\frac{1}{N D} \sum_{\nu=1}^{D} \sum_{i=1}^{N}(-1)^{\left(\Sigma_{\mu=1}^{D} i_{\mu}\right)} \tau_{i} \tau_{i+e_{\nu}},
\end{gathered}
$$

where $\left(i_{\mu} ; \mu=1, \ldots, D\right)$ are the coordinates of the point $i$ in the $D$-dimensional lattice. The main interest of defining these quantities is that they can be exactly closed in any dimension (see next section). 
Another quantity of interest is the energy response function $G_{E}\left(t, t^{\prime}\right)$ defined by

$$
G_{E}\left(t^{\prime}, t\right)=\frac{\delta m(t)}{\delta \beta\left(t^{\prime}\right)}, \quad t^{\prime}<t .
$$

This function measures the change of energy of the system at time $t$ if a small temperature change is done at $t^{\prime}<t$. Eventually we will be also interested in the integrated response function $K\left(t^{\prime}, t\right)$, defined as

$$
K\left(t^{\prime}, t\right)=\int_{t^{\prime}}^{t} G_{E}\left(t^{\prime \prime}, t\right) d t^{\prime \prime} .
$$

In order to study the response of the system to a change of temperature it will be convenient to stay in the linearresponse regime. In this regime the change in energy is linear with the variation of $\beta$. If the largest relaxation time [associated with the correlation function $\left.C_{E}\left(t^{\prime}, t\right)\right]$ is strongly dependent with the temperature and if the perturbation of $\beta$ is not too small then we can expect strong deviations from the linear-response regime. We will return to this point later.

We should note that staggered functions can be defined only in finite dimensions and have no meaning in a meanfield version of the model. Our main interest is to show that some general results on the dynamics of the 1SFM model can be inferred from the staggered one- and two-point functions even though a complete solution of its dynamics at finite temperature is still lacking.

\section{FAST PROCESSES IN FINITE DIMENSIONS}

In the finite-dimensional case some general results can be derived for the staggered magnetizations Eq. (7). In the specific case of the 1SFM the time evolution equations for $C_{0}(t), C_{1}(t)$ exactly close without the need of introducing hierarchies. Using Eq. (4) it is easy to check that the time evolution equation for $C_{0}(t)$ reads

$$
\frac{\partial C_{0}(t)}{\partial t}=-e^{-\beta} C_{0}(t) .
$$

For $C_{1}(t)$ the following result is obtained:

$$
\frac{\partial C_{1}(t)}{\partial t}=-\left(1+e^{-\beta}\right) C_{1}(t) .
$$

This simple result is a consequence of the particular dynamics for the 1SFM as defined in Eq. (4). In the case of the $2 \mathrm{SFM},{ }^{13}$ it is not clear if such a type of relation exists. Unfortunately we have not been able to generalize this closure of equations in case of higher-order correlation functions. It is important to note that staggered correlation functions are zero for homogeneous conditions (uniform or random for instance). Hence they cannot relax in time if initially they are at their equilibrium values. But it is reasonable to expect the response of the quantities $C_{0}$ and $C_{1}$ to a staggered magnetic field to be also of exponential type.

From previous equations (10) and (11) we observe the existence of at least two characteristic time scales. One of these time scales is $t_{0}=1 /\left(1+e^{-\beta}\right)$, the other one is $t_{c}=e^{\beta}$. The time scale $t_{0}$ does not diverge at zero temperature and is nearly independent of the temperature $\left(t_{0}=\frac{1}{2}\right.$ at infinite temperature and $t_{0}=1$ at zero temperature). This time scale corresponds to the relaxation time for the Ising paramagnet in absence of interaction. Hence, the time scale $t_{0}$ corresponds to the relaxation of a single spin in the presence of a heat bath and is independent of the nature of the constrained dynamics.

The time scale $t_{c}$ is more interesting. It diverges at zero temperature and has an Arrhenius behavior. Moreover, this time scale corresponds to the smallest relaxation time of one spin interacting with its nearest neighbors and is independent of the dimension of the lattice. Note that this diverging time scale is a direct consequence of the constrained dynamics in the system. Then, we conclude that $t_{c}$ is the first relevant time scale as a consequence of the cooperative phenomena which takes place in the 1SFM. We will see later that this time scale is associated with a new fast relaxation process.

In addition to these two time scales there is at least another one $t_{\text {eq }}$ associated with the large time decay of the equilibrium two-times correlation function Eq. (5). This is the largest time scale and would correspond the $\alpha$-relaxational process observed in glasses. ${ }^{15}$ Diagrammatic approximations done by Fredrickson and Andersen ${ }^{10}$ for the two-times correlation function Eq. (5) show that this time scale coincides with $t_{c}$. Our numerical investigation (in agreement with previous work ${ }^{16}$ ) suggests that $t_{\mathrm{eq}}$ is much larger than $t_{c}$ and increases with $\beta$ faster $e^{\beta}$. But more detailed investigations are necessary in order to obtain a better understanding of this point.

It is natural to think that the exponential relaxation processes described by Eqs. (10) and (11) could be present also in the relaxation of nonstaggered two-times quantities like the energy-energy correlation function Eq. (5) and the integrated response function Eq. (9). Even though we do not have a rigorous proof of this assertion we have performed Monte Carlo numerical simulations for large lattices which clearly show that this is indeed the case. In what follows, we will focus our research in the physical consequences of the fast relaxation process described by $t_{c}$. We will see the existence of a fast process of exponential character which manifests in equilibrium and off-equilibrium measurements. Unless otherwise stated, all results will refer to the 1SFM case.

\section{NUMERICAL RESULTS}

\section{A. Numerical algorithm}

We have performed Monte Carlo numerical simulations of the dynamical equations (3) in one and two dimensions. Starting from an initial configuration, the spins in the lattice are randomly selected and flipped according to the probability Eq. (3). Simulations were done for relatively large lattice sizes $N=L^{D}$ with $L=32000$ in one dimension and $L=200$ in two dimensions with periodic boundary conditions. In the range of times we are interested in (short-time processes) finite-size corrections are certainly negligible and we have checked that this is really the case. In order to test our previous exact results, Eqs. (10) and (11), we show in Fig. 1 the exponential decay of $C_{0}(t)$ at different temperatures as a function of the rescaled time $t^{\prime}=t e^{-\beta}$ in one dimension starting from the periodic condition $11101110 \ldots$. 


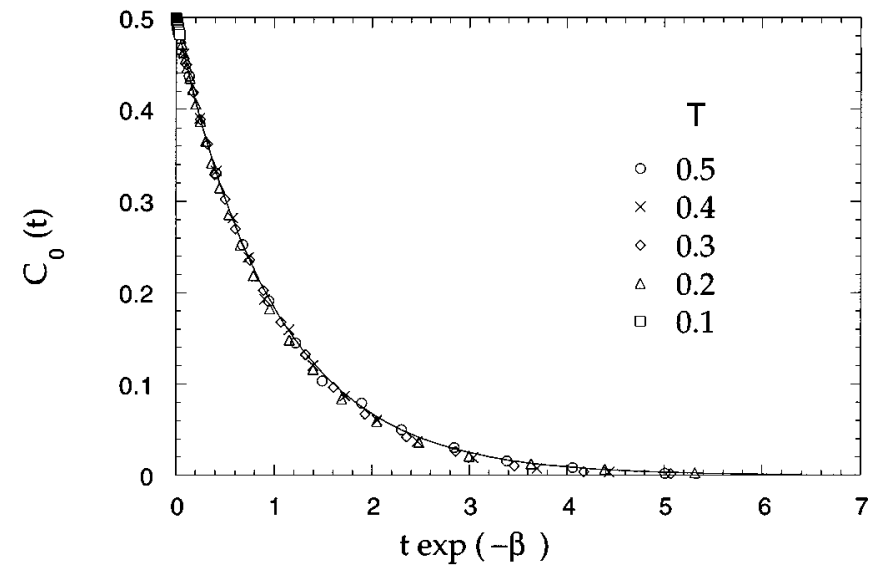

FIG. 1. Exponential relaxation of $C_{0}(t)$ in one dimension for several temperatures $T=0.1,0.2,0.3,0.4,0.5$.

\section{B. Equilibrium results}

In order to investigate the equilibrium properties, we should start from an initial fully thermalized configuration. There are several arguments which show that a dynamical transition is absent in the 1SFM in finite dimensions. ${ }^{13}$ In this case, an equilibrium configuration can be easily built because the model has no interaction in the energy function, i.e., the correlation length is zero in equilibrium. We start from a random initial configuration with energy equal to the equilibrium energy, the system is let to evolve and we compute the correlation function $C_{E}\left(t, t^{\prime}\right)$. At equilibrium, the $C_{E}\left(t, t^{\prime}\right)$ is time-translational invariant and depends only on the difference of times $C_{E}\left(t, t^{\prime}\right)=C_{E}^{\mathrm{eq}}\left(t-t^{\prime}\right)$. We have carefully checked this point. We have repeated these measurements at different temperatures. We have observed that correlation functions display two regimes separated by the critical time $t_{c}=e^{\beta}$. In the first initial regime (for times smaller than the critical time $t_{c}=e^{\beta}$ ) correlation functions decay exponentially fast. Empirically we find that the relaxation time associated with this exponential process is (with very high precision) $2 t_{c}$,

$$
C_{E}^{\mathrm{eq}}(t) \simeq \exp \left(-t /\left(2 t_{c}\right)\right)=\left(C_{0}(t)\right)^{1 / 2} ; \quad t \ll t_{c} .
$$

In the second regime, for times larger than $t_{c}$, the relaxation turns out to be much slower with stretched-exponential behavior at high temperatures which comes close to a powerlaw behavior at low temperatures. We think that no conclusive relaxation behavior can be guessed numerically in this second regime. A crude estimate of the relaxation time $t_{\text {eq }}$ for the second regime can be obtained (integrating the equilibrium correlation function respect to the time) and we obtain values larger than $t_{c}$. This is an interesting point which deserves a more detailed investigation and should yield the relaxation time $t_{\text {eq }}$ for the slowest relaxational process. ${ }^{17}$ Figure 2 shows $C_{E}^{\mathrm{eq}}(t)$ in one dimension at three different temperatures $T=0.15,0.2,0.40$. The values of the critical time associated with these temperatures are $t_{c} \simeq 780,150,12$, respectively. These critical times are indicated with an arrow in the figure. The initial exponential decay Eq. (12) is also depicted in the figure as a continuous line.

Similar results have been obtained in two dimensions where we expect the this fast process to be independent on

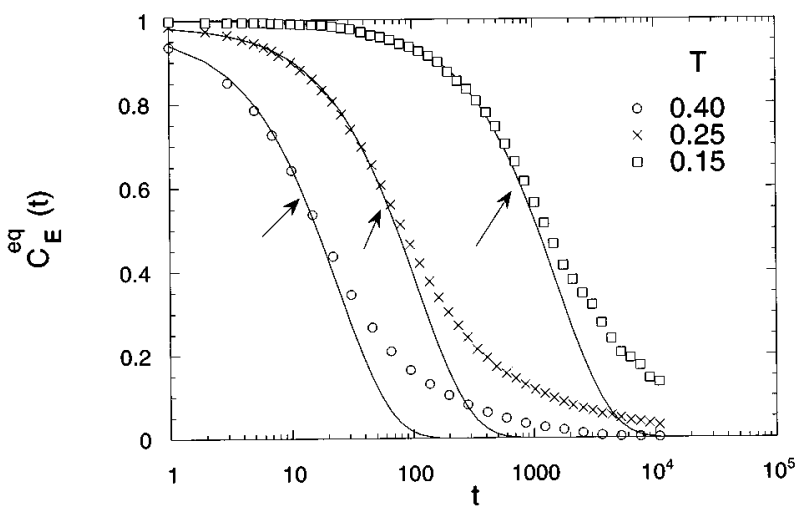

FIG. 2. $C_{E}^{\mathrm{eq}}(t)$ in one dimension at temperatures $T=0.15,0.25,0.4$. The continuous lines are the exponential relaxations, Eq. ( 12).

the dimension. This is shown in Fig. 3 where we plot the $C_{E}^{\mathrm{eq}}(t)$ for two temperatures $T=0.15,0.25$ in one and two dimensions and the expected relaxation Eq. (12). The values of the critical time associated with these temperatures are $t_{c} \simeq 780,55$, respectively. These times are indicated with an arrow in the figure. From this figure it can be clearly appreciated that the regime $t<t_{c}$ (the region of small times limited by the arrow) is independent of the dimension of the system as argued previously. The empirical relaxation Eq. (7) which fits pretty well the fast decay process is also depicted by a continuous line. In the second regime $t>t_{c}$ relaxation becomes dependent on the dimension and slower in one dimension than in two dimensions as expected (dynamical constraints in the 1SFM tend to forbid less paths in phase space as the dimensionality of the lattice increases).

We have also analyzed the integrated response function at equilibrium $K\left(t^{\prime}, t\right)=K^{\mathrm{eq}}\left(t-t^{\prime}\right)$. In order to measure it we have prepared the system at equilibrium at a temperature $\beta$. After some time we change the temperature of the system by the quantity $\Delta \beta$ and we let the system evolve at the new constant temperature $\beta+\Delta \beta$. If the change of temperature was applied at zero time then we have

$$
K^{\mathrm{eq}}(t)=\frac{m_{\beta+\Delta \beta}(t)-m_{\beta}^{\mathrm{eq}}}{\Delta \beta} .
$$

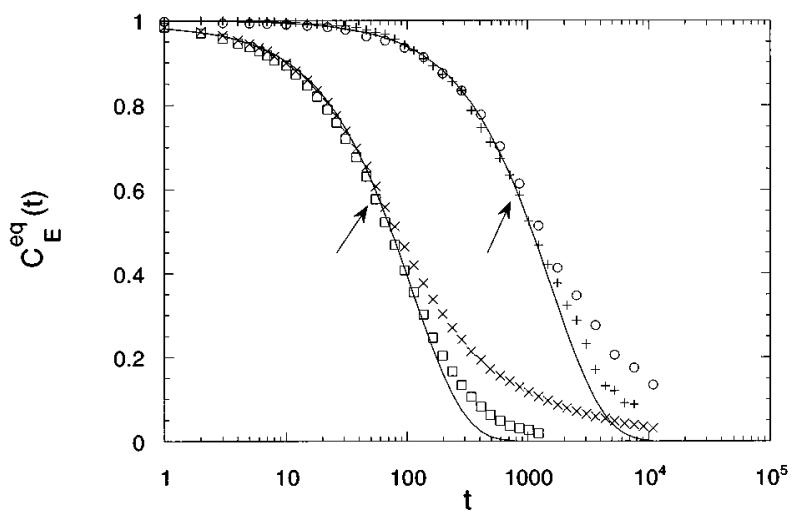

FIG. 3. $C_{E}^{\mathrm{eq}}(t)$ at temperatures $T=0.15[D=2$ (squares), $D=1$ (crosses) $] T=0.25[D=2$ (times), $D=1$ (circles) $]$ in one and two dimensions. The continuous lines are the exponential relaxations, Eq. (12). 


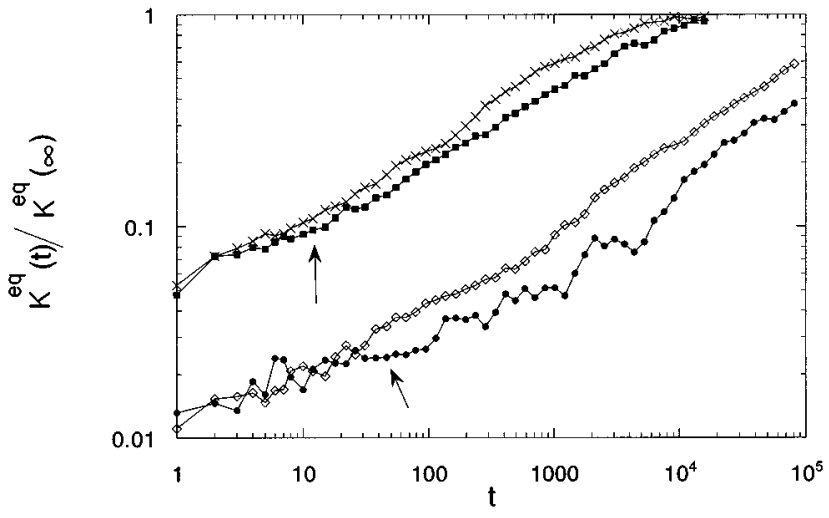

FIG. 4. Normalized $K^{\mathrm{eq}}(t)$ in one dimension at temperatures $T=0.4$ with $\Delta \beta=0.2$ (squares), -0.2 (crosses), and $T=0.25$ with $\Delta \beta=0.4$ (rhombs), -0.4 (dots).

In order to be in the linear-response regime it is necessary to make the change $\Delta \beta$ small enough. But it is important to note that $\Delta \beta$ cannot be arbitrarily small, otherwise the response of the system to the temperature change is very small and the $K^{\mathrm{eq}}(t)$ measurements become much too noisy.

In Fig. 4 we plot the $K^{\mathrm{eq}}(t)$ normalized to its infinite time equilibrium value $K^{\mathrm{eq}}(\infty)$. This quantity converges to 1 in the infinite time limit. Numerical experiments are shown in one dimension at two different temperatures $T=0.4$ and $T=0.25$ (data have been averaged over 20 different runs), for two different signs of the perturbation $\Delta \beta$. The results of the figure display some deviations from the linear-response regime especially at large times and low temperatures. As commented previously, the equilibrium relaxation time $t_{\mathrm{eq}}$ is strongly dependent on the temperature and increases with $\beta$ faster than does $t_{c}$. Then we expect the response of the system to a perturbation of the temperature to display departures from linearity in the part of the relaxation process controlled by the time scale $t_{\text {eq }}$. In the part of the relaxation process dominated by $t_{c}$ this effect should be smaller. Support in favor of this argument is shown in Fig. 4 where the arrow indicates the value of the critical time for the two temperatures. The dependence on the sign of the perturbation seems to be stronger for the slowest part of the relaxation (i.e., for times $t>t_{c}$ ) than in the fast part of the relaxation (i.e., for times $t<t_{c}$ ). In order to get more clear cut results it would be necessary to go to lower temperatures. Unfortunately the $K^{\mathrm{eq}}(t)$ becomes much too noisy even though we have been able to confirm this trend.

\section{Off-equilibrium results}

In this section we want to investigate the role of the critical time $t_{c}$ for nonequilibrium relaxation processes. We are going to show that $t_{c}$ sets a minimum time scale above which nonequilibrium relaxations display aging effects. In the following we will use the notation in which $t^{\prime} \rightarrow t_{w}, t \rightarrow t_{w}+t$ ( $t_{w}$ stands for waiting time). We have done numerical simulations of the 1SFM in one and two dimensions measuring $C_{E}\left(t_{w}, t_{w}+t\right)$.

Results for the $C_{E}\left(t_{w}, t_{w}+t\right)$ are shown in Fig. 5 in the one-dimensional case. We start from a random initial condition $^{19}$ with initial energy $E(t=0)=-0.5$ quite far from

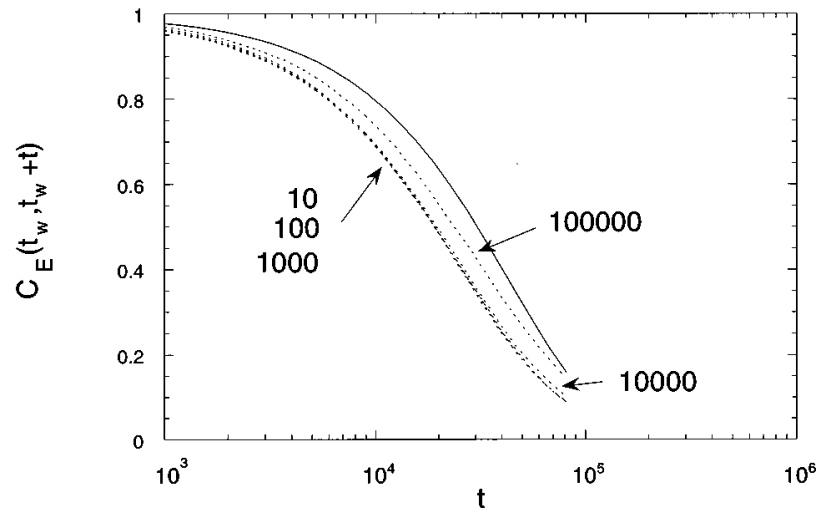

FIG. 5. $C_{E}\left(t_{w}, t_{w}+t\right)$ for different waiting times $t_{w}=10,10^{2}, 10^{3}, 10^{4}, 10^{5}$ in one dimension at $T=0.1$. For $t_{w}=10,10^{2}, 10^{3}$ the relaxation curves superimpose and aging is absent.

its equilibrium value $\left[E^{\mathrm{eq}}(\beta)=-1 /\left(1+e^{-\beta}\right)\right]$. We computed the $C_{E}\left(t_{w}, t_{w}+t\right)$ for different values of the waiting time $t_{w}=10,10^{2}, 10^{3}, 10^{4}, 10^{5}$ at $T=0.1$ (at this temperature $t_{c} \simeq 22000$ ) in one and two dimensions (we show only the results in one dimension, in two dimensions they are qualitatively similar). In order to clearly appreciate the qualitative trend of the data we only show the values of $t$ within the range $10^{3}-10^{5} \mathrm{MCS}$. Figure 5 shows two regimes. In the first regime $\left(t_{w}=10,10^{2}, 10^{3}<t_{c}\right)$ aging effects are absent, i.e., the relaxation curve $C_{E}\left(t_{w}, t_{w}+t\right)$ only depends on $t$. In the regime $t_{w}=10^{5}>t_{c}$ aging effects appear [i.e., the full relaxation curve $C_{E}\left(t_{w}, t_{w}+t\right)$ also depends on $\left.t_{w}\right]$. The continuous line is the equilibrium exponential behavior Eq. (12).

As emerges from Fig. 5, the aging effects in the correlation function are very small even for $t_{w}>t_{c}$ at least in the region of times where the value of the correlation function is not too small. In fact, a scaling of the type $C_{E}\left(t_{w}, t_{w}+t\right)=f\left(t / t_{w}\right)$ will not work at all. This is surprising since one would expect in the off-equilibrium slow regime a dynamical behavior plagued of strong aging effects in the correlation function. Possibly, this is a consequence of the particular correlation function used. In what follows we will see that the response function displays clear aging effects.

We have measured the off-equilibrium integrated response function starting from a random initial configuration. The system is let to evolve for a time $t_{w}$ at constant (inverse temperature) $\beta$. At time $t_{w}$ we make a copy of the system and we make it evolve at the constant new temperature $\beta+\Delta \beta$. After $t_{w}$ we measure the difference between the magnetizations of the two copies evolving with the same thermal noise but different temperatures. In this way we compute

$$
K\left(t_{w}, t_{w}+t\right)=\frac{m_{\beta+\Delta \beta}\left(t+t_{w}\right)-m_{\beta}\left(t+t_{w}\right)}{\Delta \beta} .
$$

In Fig. 6 we show the normalized integrated response function $K\left(t_{w}, t_{w}+t\right) / K^{\mathrm{eq}}(\infty)$ at $T=0.1\left(t_{c} \simeq 22000\right)$ for different values of $t_{w}=10,10^{2}, 10^{3}, 10^{4}, 10^{5}$ in the one-dimensional case. We can clearly appreciate the existence of the critical time $t_{c}$ which separates a regime where there is 


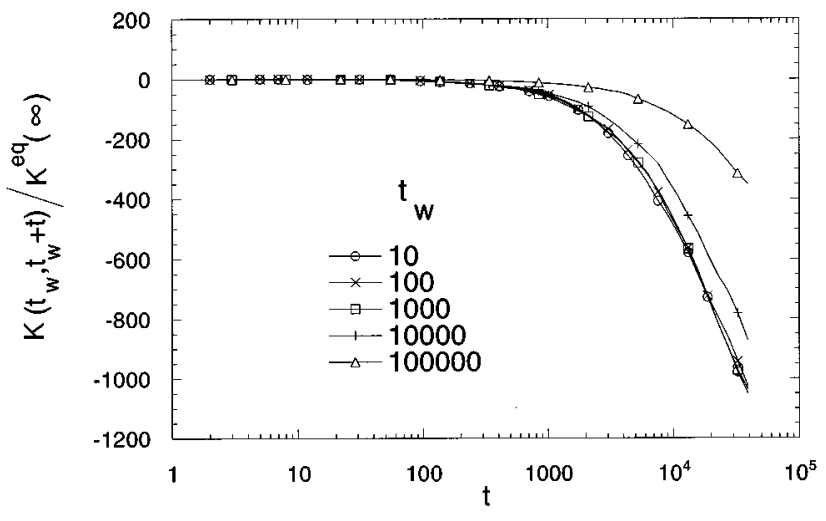

FIG. 6. Normalized $K\left(t_{w}, t_{w}+t\right)$ at $T=0.1$ for several values of $t_{w}$ and $\Delta \beta=0.5$. The symbols are a guide to the eyes. Note that $K^{\mathrm{eq}}(t)$ is nearly zero in this range of times.

no $t_{w}$ dependence from a regime where the system ages. As $t_{w}$ increases the response of the system becomes smaller which is a typical feature of aging. ${ }^{20}$ Note that the equilibrium value of $K^{\mathrm{eq}}(t)$ in the window of time shown in the Fig. 6 is practically zero (and converges to 1 for large times) while in the off-equilibrium regime the response reaches a (negative) approximate value of $-10^{3}$. Hence the response of the system is three orders of magnitude larger and negative in the off-equilibrium regime than in the equilibrium case.

\section{CONCLUSIONS}

This work has been devoted to the study of one of the simplest constrained kinetic Ising models, i.e., the so-called $n$ SFM introduced by Friedrickson and Andersen in the special case of $n=1$. This is an interesting model with an extremely simple energy function where the frustration is contained in the dynamical rules which forbid certain transitions between configurations in phase space.

Our main interest has been the research of fast processes in the 1SFM. In this model the rate of variation of the energy in one point of the lattice is linearly coupled to the energy of the nearest neighbors. This is the simplest case one can consider, while the 2SFM corresponds to a quadratic coupling between the nearest-neighbor sites in the lattice. ${ }^{10}$ In case of the 1SFM some exact closed dynamical equations can be obtained for the one- and two-point staggered functions $C_{0}$ and $C_{1}$ in any dimensions. This reveals that both staggered functions $C_{0}$ and $C_{1}$ decay exponentially fast (with characteristic times $t_{c}$ and $t_{0}$ ) independently of the dimensionality of the lattice. $t_{0}$ is the relaxation of a single spin uncoupled to its nearest neighbors embedded in a thermal bath (hence, independent of the dimension) and $t_{c}$ is an Arrhenius temperature-dependent relaxation time resulting from the dynamical constraints. Then we expect the existence of these fast processes in other nonstaggered quantities like the energy-energy correlation function and also the integrated response function. While we have not found a precise demonstration of this result we have given strong numerical support to this hypothesis by measuring the equilibrium and nonequilibrium behavior of the correlation function Eq. (5) and integrated response function Eq. (9) in one- and two- dimensional lattices. In the equilibrium case the correlation function Eq. (5) decays exponentially fast with an (empirically found) relaxation time $2 t_{c}$ in the regime of times $t<t_{c}$ while relaxation becomes slower in the regime $t>t_{c}$. Furthermore, the integrated response function displays strong nonlinear effects in the regime $t>t_{c}$ specially at low temperatures. Concerning the off-equilibrium behavior we find that aging effects are absent for values of the waiting time less than the critical time. This is nicely observed in the behavior of the integrated response function (Fig. 6).

Now we should discuss to what extent our results are general and not confined to the particular 1SFM case. Equations (10) and (11) are exact results in the 1SFM case and we expect they are not more valid in the $n$ SFM with $n$ larger than 1 . We have performed some numerical simulations in case of the 2SFM but we have not found evidence on the existence of this critical time. This could explain why some previous numerical works on the 2SFM (Ref. 13) were able to fit the equilibrium relaxation functions to a stretchedexponential behavior, while in the 1SFM this is not possible due to the existence of two different regimes. In numerical simulations one is able to explore only relatively small scales of time. It is clear that an initial exponential process would make the numerical fits of the slow regime difficult, specially in the low-temperature phase where the critical time starts to become large. Consequently, the nature of the slow relaxational processes in the one-spin facilitated Ising model is difficult to establish using numerical simulations. This is a necessary task to estimate the relaxation time for the slow processes $t_{\text {eq }}$ in this model in order to draw conclusions about the fragility of the glass.

Also in the realm of disordered systems (for instance in spin glasses ${ }^{7}$ ) we are not aware of the existence of this critical time, possibly because in that case frustration is directly introduced in the energy function and not in the dynamics. We are tempted to conclude that (1) the existence of a temperature activated critical time and (2) the presence of exponential decay processes in the relaxation of some correlation functions in the short-time regime, are both strictly related to the nature of this type of constrained short-range dynamics.

Summarizing, we have found evidence about the existence of two fast processes in the 1SFM model. The first process with characteristic time $t_{0}$ corresponds to the relaxation of single spin in the lattice. This is a trivial process not related to any cooperative effect in the lattice. The second process is a fast exponential process consequence of the dynamical constraints. Physically it would correspond to the relaxation of some coupled degrees of freedom of the system. The results which emerge from the study of the 1SFM are in agreement with some recent experimental findings by Colmenero et al. on fragile polymer glasses. By doing neutron-scattering measurements in glasses they claim the existence of a temperature-activated critical time (with small energy barrier) which separates two well-defined time regions. In the first region the relaxation is exponential, while in the second regime relaxation is much slower with higher relaxation time. This feature seems to be captured by the present model.

It would be very interesting to analytically solve the dynamics at finite temperature of this model (at least in one dimension) in order to confirm the numerical findings of this 
work. This would also shed light on the existence of other relaxational processes (like the $\alpha$ and $\beta$ relaxation) as predicted by the mode coupling theory.

\section{ACKNOWLEDGMENTS}

F.R. acknowledges Ministerio de Educacion y Ciencia of Spain for financial support. E.F. acknowledges Gobierno de Navarra for financial support through a predoctoral grant. We are grateful to Vicente Azcoiti and Paco Padilla for a careful reading of the manuscript.

\section{APPENDIX}

In this appendix we present the exact solution of the 1SFM in one dimension at zero temperature. Let us take a chain of $N$ spins $\sigma_{i}$ and we define the new set of variables $\tau_{i}=1-\sigma_{i}$. In terms of this set of variables we define the following set of correlation functions:

$$
D_{k}(t)=\frac{1}{N} \sum_{r=1}^{N} \tau_{r}(t) \tau_{r+1}(t) \ldots \tau_{r+k}(t) .
$$

The magnetization $m_{\tau}=(1 / N) \sum_{r=1}^{N} \tau_{r}=D_{0}(t)$ is the first term of this hierarchy. Using Eq. (4) we can derive the time evolution of the $D_{k}(t)$ at zero temperature. We get

$$
\frac{\partial D_{k}}{\partial t}=-D_{k+1}-k D_{k} ; \quad k \geqslant 0 .
$$

We introduce the generating function

$$
G(x, t)=\sum_{k=1}^{\infty} \frac{x^{k}}{k !} D_{k}(t) .
$$

In terms of this generating function we have the following partial differential equation:

$$
\frac{\partial G(x, t)}{\partial t}=-(1+x) \frac{\partial G(x, t)}{\partial x} .
$$

This linear partial differential equation is readily solved yielding

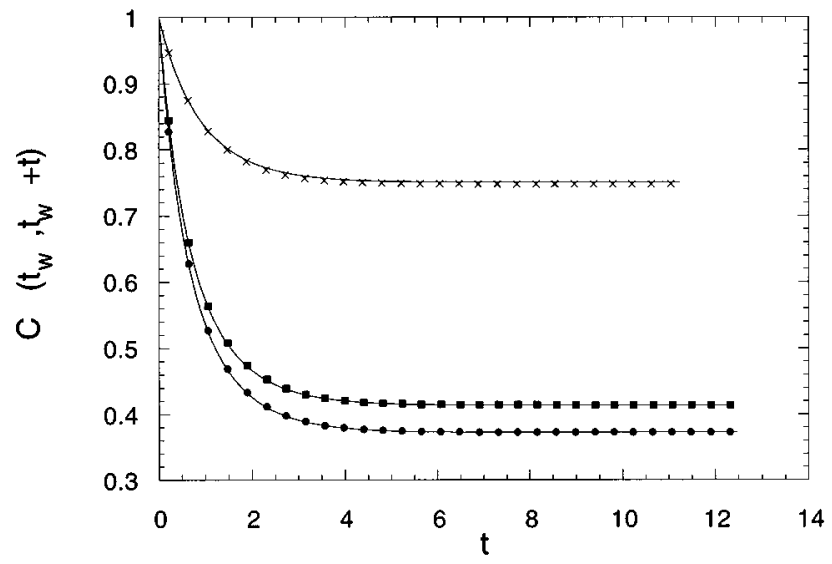

FIG. 7. $C_{E}\left(t_{w}, t_{w}+t\right)$ for different waiting times $t_{w}=0.0125,0.125,1.25$ at zero temperature in one dimension. The lines are the exact solution (A7).

$$
G(x, t)=G_{0}\left[(1+x) e^{-t}-1\right],
$$

where $G_{0}(x)=G(x, 0)$ is the initial condition. We can obtain the different set of moments

$$
D_{k}=\left(\frac{\partial^{k} G}{\partial x^{k}}\right)_{x=0}
$$

In particular we get for the magnetization $m_{\tau}=G_{0}[\exp (-t)-1]$. In the large time limit it converges to $G_{0}(-1)$ depending on the initial condition. For the particular initial condition $\sigma_{i}=0, G_{0}(x)=e^{x}$ and the magnetization $m_{\tau}$ does not converge to its equilibrium value $\left(m_{\tau}^{\mathrm{eq}}=1\right)$ but to $1 / e$.

We can also compute the two-times correlation function Eq. (5). At zero temperature we find

$$
C_{E}\left(t^{\prime}, t\right)=\frac{1-m(t)}{1-m\left(t^{\prime}\right)}=\frac{m_{\tau}(t)}{m_{\tau}\left(t^{\prime}\right)}=\frac{G_{0}\left(e^{-t}-1\right)}{G_{0}\left(e^{-t^{\prime}}-1\right)} .
$$

In Fig. 7 we compare Eq. (A7) with the numerical results for different values of $t^{\prime}$.
${ }^{1}$ W. Gotze, in Liquid, Freezing and the Glass Transition, 1989 Les Houches Lectures, edited by J. P. Hansen, D. Levesque, and J. Zinn-Justin (North-Holland, Amsterdam, 1989); C. A. Angell, Science 267, 1924 (1995).

${ }^{2}$ E. Lentheusser, Phys. Rev. A 29, 2765 (1984); T. R. Kirkpatrick, ibid. 31, 939 (1985); W. Gotze and L. Sjogren, Rep. Prog. Phys. 55, 241 (1992).

${ }^{3}$ F. Fujara and W. Petry, Europhys. Lett. 4, 571 (1987); B. Frick and D. Richter, Phys. Rev. B 47, 14795 (1993).

${ }^{4}$ J. Colmenero, A. Arbe, and A. Alegria, Phys. Rev. Lett. 71, 2603 (1993).

${ }^{5}$ S. Franz and G. Parisi, J. Phys. (France) I 5, 1401 (1995).

${ }^{6}$ F. Ritort, Phys. Rev. Lett. 75, 1190 (1995); A. Barrat and M. Mezard, J. Phys. (France) I 2, 705 (1992).

${ }^{7}$ M. Mézard, G. Parisi, and M. A. Virasoro, Spin Glass Theory and Beyond (World Scientific, Singapore, 1987); K. H. Fischer and
J. A. Hertz, Spin Glasses (Cambridge University Press, Cambridge, 1991).

${ }^{8}$ T. R. Kirkpatrick and D. Thirumalai, J. Phys. A 22, L149 (1989); J. P. Bouchaud and M. Mezard, J. Phys. (France) I 4, 1109 (1994); E. Marinari, G. Parisi, and F. Ritort, J. Phys. A 27, 7615 (1994); 27, 7647 (1994).

${ }^{9}$ R. G. Palmer, in Lectures in the Science of Complexity, edited by D. L. Stein (Addison-Wesley, Reading, MA, 1989), and references therein; G. H. Fredrickson, Annu. Rev. Phys. Chem. 39, 149 (1988).

${ }^{10}$ G. H. Fredrickson and H. C. Andersen, Phys. Rev. Lett. 53, 1244 (1984).

${ }^{11}$ Note that at infinite temperature the system performs a nontrivial random walk in phase space.

${ }^{12}$ S. Franz and J. Hertz, Phys. Rev. Lett. 74, 2114 (1995); J. P. Bouchaud, L. F. Cugliandolo, J. Kurchan, and M. Mezard, 
Physica A (to be published); J. P. Bouchaud, A. Comtet, and C. Monthus, J. Phys. (France) I 5, 1521 (1995).

${ }^{13}$ G. H. Fredrickson and H. C. Andersen, J. Chem. Phys. 83, 5822 (1985); G. H. Fredrickson and S. A. Brawer, ibid. 84, 3351 (1986)

${ }^{14}$ The same correlation function is obtained in terms of the variables $\left\{\sigma_{i} ; i=1, \ldots, N\right\}$.

${ }^{15}$ Whether in the 1 SFM there is also the $\beta$ relaxational processes as predicted by the mode coupling theory (Ref. 1) is still unclear.

${ }^{16}$ M. J. Ruiz-Montero, Ph.D. thesis, University of Sevilla, 1992; J. J. Brey and M. J. Ruiz-Montero, Phys. Rev. B 43, 585 (1991).
${ }^{17}$ Stretched-exponential behavior already appears in the Glauber dynamics of the ferromagnetic one-dimensional Ising model. In this case it is difficult to guess the correct relaxational behavior from numerics. See the discussion of Brey and Prados on this point (Ref. 18).

${ }^{18}$ J. J. Brey and A. Prados, Phys. Rev. E 53, 458 (1996).

${ }^{19}$ The same results were obtained starting from a periodic initial configuration like for instance $11101110 \ldots$ where $C_{0}(t=0)$ and $C_{1}(t=0)$ are different from zero.

${ }^{20}$ J. P. Bouchaud, J. Phys. (France) I 2, 1705 (1992); L. F. Cugliandolo and J. Kurchan, Philos. Mag. B 71, 50 (1995). 